\title{
Heat transfer effects on a fully premixed methane impinging flame
}

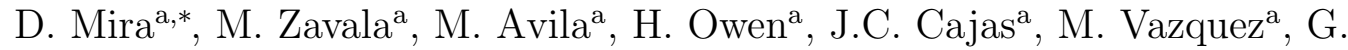
Houzeaux $^{\mathrm{a}}$

${ }^{a}$ CASE Department, Barcelona Supercomputing Center (BSC-CNS), Barcelona, Spain

\begin{abstract}
A numerical assessment of different thermal conditions for an impinging flame configuration is investigated using large-eddy simulation. The cases of study correspond to a turbulent methane flame at equivalence ratio $\mathrm{ER}=0.8$ and temperature $\mathrm{T}=298 \mathrm{~K}$ exiting at $30 \mathrm{~m} / \mathrm{s}$ with a nozzle-to-plate distance over diameter of $\mathrm{H} / \mathrm{D}=2$. Computational cases based on different thermal conditions are compared to a conjugate case, in which fluid and solid domains are solved simultaneously. A solid material defined with enhanced conductivity properties is used to represent the wall in the conjugate case, so that the characteristic time scales of the solid are reduced. The results indicate that the heat transfer condition influences not only the mean temperature and gradients, but also the temperature fluctuations in the near-wall region. It is found that adiabatic, isothermal and more sophisticated Robin-type boundary conditions contribute to underpredict/overpredict the temperature field near the wall. As the time scales of fluid and solid are of the same order, the use of conjugate approaches is required to predict the correct flow fields near the wall and the skin temperature.
\end{abstract}

Keywords: Conjugate heat transfer (CHT), impinging flame, large-eddy simulation, thermal boundary conditions

\section{Introduction}

The effects of heat transfer on engineering applications is a fundamental aspect in the design of power and propulsive systems. The heat exchange between fluid and solid components can reduce the system efficiency in situations where temperature peaks and gradients become important. This occurs in applications such as gas turbines or combustion engines, in which the cooling is essential to reduce wall tem-

\footnotetext{
${ }^{*}$ Corresponding author: daniel.mira@bsc.es (D. Mira)

Phone number: +34934054286
} 
perature below the melting point. Heat losses usually carry a reduction in efficiency that eventually might lead to an economical cost and potential failure [1]. However, this interaction is difficult to model numerically not only due to the different time scales governing each individual problem, but also due to instability issues related to the coupling strategy $[2,3]$.

The coupling of the Navier-Stokes equations with the heat equation, also referred as conjugate heat transfer, usually requires the existence of two different solvers, one for each domain, exchanging information at specified boundaries. Partitioned or staggered algorithms can be applied to control the exchange of information allowing the solvers to advance at different time-steps with the most suitable formulation for each physical problem [4]. In the context of Reynolds-averaged Navier-Stokes (RANS) applications, the fluid and solid domain can be converged separately and then exchange the heat fluxes and skin temperatures. When only the stationary solution is sought, this process is repeated until the full convergence of the two physical problems [5, 6, 7]. More complex approaches in the RANS context have been proposed by Craft et al. [8] using transport equations for temperature variance and its dissipation rate across the solid wall region.

Only recent work can be found related to conjugate heat transfer problems in the context of large-eddy simulation (LES). The work of Chatelain et al. 9] addresses the evolution of temperature fluctuations within a solid material when different wall boundary conditions are used in the fluid domain for a plane channel flow. Different coupling strategies for the conjugate problem in LES were examined by Duchaine et al. [7] in terms of exchange quantities and stability using a cooled turbine blade model. Maheu et al. 10, also investigated the heat transfer effects of a low-Mach turbine blade using LES and the same methodology was applied to a practical aeronautical combustor in the work of Jaure et al. [1].

An interesting application to evaluate the numerical algorithms and the effects of thermal coupling with the flow dynamics is the case of a jet flame impinging on a plate. In this configuration, high rates of wall heat transfer are achieved as a consequence of the enhancement of the heat transfer coefficients by the continuous impact of vortical structures on the plate [11]. Impinging jets are characterised by the ratio $\mathrm{H} / \mathrm{D}$, where $\mathrm{H}$ is the nozzle-to-plate distance and $\mathrm{D}$ is the flow inlet diameter. Three flow regions can be identified in this configuration: the free jet region where flow is not influenced by the plate, the deflection region and the wall jet region [12]. For large H/D ratios, the configuration permits the development of a free jet and the impingement leads to the formation of a heat transfer profile with a maximum located at the stagnation point. However, for small H/D ratios, the jet core may be longer than the $\mathrm{H} / \mathrm{D}$ ratio causing the jet to be laminar at the plate featuring low levels of turbulence and a minimum heat transfer coefficients at the stagnation point. As the flow accelerates parallel to the plate, the wall shear stresses increase leading to a maximum in the heat transfer profile at the wall [13]. 
Numerical studies on impinging jets have shown that traditional RANS models are incapable of predicting the main flow dynamics, and unsteady techniques such as LES or direct-numerical simulation (DNS) have been used instead providing better predictions [14, 15]. LES studies on non-reacting impinging jets have been focused on the effects of subgrid scale modelling [14, 16, inlet conditions or flow dynamics [13] and formation of vortical structures [12]. In case of reacting flows, impinging flames have also been investigated in the literature. The studies mainly focus on flame dynamics and near-wall behaviour [17], effects of wall impingement and swirling motion [18, fuel variability and chemical kinetics [11, 19], combustion heat losses using tabulated chemistry [20] among others. These studies are focused on flame dynamics and little interest is given to the thermal boundary conditions imposed at the impinging wall.

The present work addresses the effect of the heat transfer condition at the solid wall on a premixed impinging jet flame configuration at $\mathrm{H} / \mathrm{D}=2$. Different thermal conditions are imposed at the impinging plate and its effects on the flame dynamics, heat transfer enhancement, shear stress and wall-jet development are discussed in the context of LES.

\section{Mathematical formulation}

The governing equations describing the reacting flow field correspond to the low Mach number approximation of the Navier-Stokes equations along with the species conservation equations [21. The flow field is filtered in space using a box filter given by $\Delta=V^{1 / 3}$, where $V$ represents the cell volume and a Favre filter operator is employed to account for density variations. The filtered governing equations for multi-species reacting flows for LES are:

$$
\begin{gathered}
\frac{\partial \bar{\rho}}{\partial t}+\frac{\partial\left(\bar{\rho} \widetilde{u}_{j}\right)}{\partial x_{j}}=0 \\
\frac{\partial\left(\bar{\rho} \widetilde{u}_{i}\right)}{\partial t}+\frac{\partial\left(\bar{\rho} \widetilde{u}_{j} \widetilde{u}_{i}\right)}{\partial x_{j}}=-\frac{\partial \bar{p}}{\partial x_{i}}+\frac{\partial \bar{\tau}_{i j}}{\partial x_{j}}-\frac{\partial \tau_{i j}^{s g s}}{\partial x_{j}} \\
\frac{\partial\left(\overline{\rho c}_{p} \widetilde{T}\right)}{\partial t}+\frac{\partial\left(\overline{\rho c}_{p} \widetilde{u}_{j} \widetilde{T}\right)}{\partial x_{j}}=\frac{D p^{t h}}{D t}+\frac{\partial}{\partial x_{j}}\left(\bar{\lambda} \frac{\partial \widetilde{T}}{\partial x_{j}}\right)+\left(\bar{\rho} \sum_{k=1}^{N} \bar{c}_{p, k} \bar{D}_{k} \frac{\partial \widetilde{Y}_{k}}{\partial x_{j}}\right) \frac{\partial \widetilde{T}}{\partial x_{j}}+ \\
\frac{\partial\left(\bar{\rho} \widetilde{Y}_{k}\right)}{\partial t}+\frac{\partial\left(\widetilde{u}_{i}\right.}{\partial x_{j}}+\frac{\overline{Q^{c}}}{\partial x_{j}}-\frac{\left.\partial \widetilde{Y}_{j}\right)}{\partial x_{j}}=\frac{\partial}{\partial x_{j}}\left(\bar{\rho} \bar{D}_{k} \frac{\partial \widetilde{Y}_{k}}{\partial x_{j}}\right)+\overline{\dot{\omega}}_{k}-\frac{\partial \Phi_{j, k}^{s g s}}{\partial x_{j}} \quad k=1, \ldots, N
\end{gathered}
$$


where $t, x_{i}, \bar{\rho}, \widetilde{u}_{i}, \bar{p}, \bar{\tau}_{i j}, \widetilde{T}, p^{t h}$ and $\widetilde{Y}_{k}$ are the time, $i$ th direction with $\mathrm{i}, \mathrm{j}=1,2,3$, density, $i$ th velocity component, pressure, viscous stress tensor, temperature, thermodynamic pressure and species mass fraction respectively. The thermodynamic pressure $p^{\text {th }}$ is set to the ambient pressure $(1$ bar) and remains constant for open flows. The thermal conductivity of the mixture, the enthalpy and the specific heat of each species $k$ are defined as $\bar{\lambda}, \bar{h}_{k}$ and $\bar{c}_{p, k}$. The heat release rate $\bar{Q}^{c}$ is defined as:

$$
\overline{\dot{Q}^{c}}=-\sum_{k}^{N}\left(h_{k}+\Delta h_{f, k}^{0}\right) \overline{\dot{\omega}}_{k}
$$

The production rate of the $k$ th species $\overline{\dot{\omega}}_{k}$ is obtained as a summation of all reactions involving species $k$ and is expressed using an Arrhenius form [21]. The index $k=(1$ to $N$ ) denotes the individual species, while the superscript $s g s$ refers to the subgrid scale terms coming from the filtering operation. The variables with superscript sgs correspond to the unresolved momentum transport $\tau_{i j}^{s g s}=\bar{\rho}\left(\widetilde{u_{i} u_{j}}-\widetilde{u}_{i} \widetilde{u}_{j}\right)$, the unresolved heat flux $h^{s g s}=\bar{\rho}\left(\widetilde{u_{j} T}-\widetilde{u}_{j} \widetilde{T}\right)$ and the unresolved species mass flux $\Phi_{j, k}^{s g s}=\bar{\rho}\left(\widetilde{u_{j} Y_{k}}-\widetilde{u}_{j} \widetilde{Y}_{k}\right)$ respectively. The subgrid scale momentum transport is modelled using an eddy viscosity $\nu_{t}$ obtained by the Wall-Adapting Local Eddyviscosity (WALE) model proposed by Nicoud and Ducros [22]. The heat flux in the subgrid scale $h^{s g s}$ as well as the subgrid scale species mass flux $\Phi_{k}^{s g s}$ are both modelled using a gradient diffusion approach [23].

The solid part of the domain, when solved, is treated by the conjugate heat transfer (CHT) approach, for which the heat conduction equation is solved in the solid domain. The equation reads:

$$
\rho_{s} c_{p} \frac{\partial T}{\partial t}=\frac{\partial}{\partial x_{j}}\left(k_{s} \frac{\partial T}{\partial x_{j}}\right)+g
$$

where $\rho_{s}, k_{s}$ and $g$ are the density of the solid material, the conductivity of the solid and the external heat source respectively.

\subsection{Coupling approach for the LES-CHT simulation}

The coupling of the solid domain with the LES solver is accomplished by imposing the heat flux from the fluid into the solid domain, while the solid imposes the skin temperature $\mathrm{T}_{s}$ into the fluid domain. The boundary conditions are set at the interface plane between the solid and fluid domains (see Fig. 1 (a)) using the nature of the information received by each solver. The heat equation Eq. (6) requires a Neumann-type boundary condition $\mathrm{q}=\mathrm{q}_{s}$ at the interface, while the Low-Mach fluid equation Eq. (3) uses a Dirichlet-type boundary condition for the temperature $\mathrm{T}=\mathrm{T}_{s}$ [24]. The coupling of the solid and fluid meshes is shown in Fig. 1 (b), 
where the nodes of the solid domain are represented by thick dots on the interface of the fluid domain. Despite no matching is required for the coupling algorithm, the meshes were set coincident at the interface to avoid interpolation errors.

An adaptation of the Parallel Location and Exchange library (PLE) [25] intended to provide mesh or particle-based code coupling services is employed for the LESCHT coupling. It allows an easy communication between application codes written in $\mathrm{C} / \mathrm{C}++$, Fortran or Python that are running and share the same communicator. This communicator is split into one communicator for the execution of each solver (fluid and solid). The communication between the applications takes place using the intracommunicators created after splitting the global communicator.

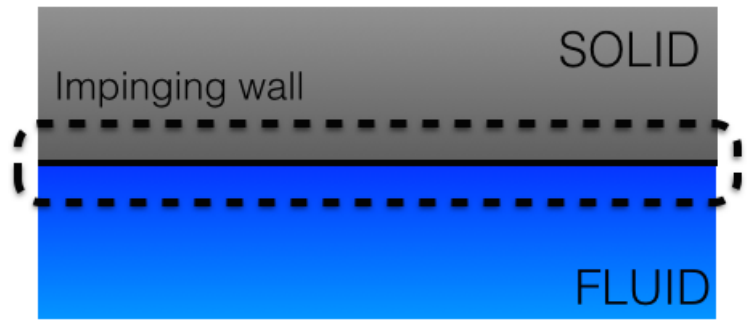

(a)

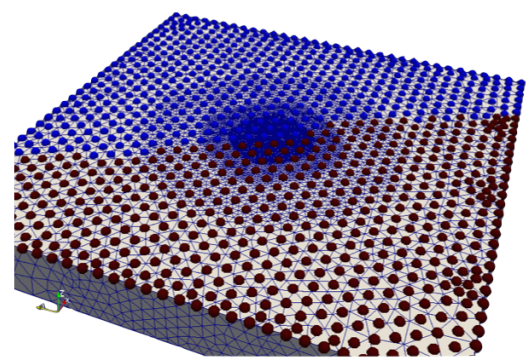

(b)

Figure 1: Interface between solid and fluid domain (a) and matching between fluid and solid meshes (b).

\subsection{Chemical kinetics and turbulent combustion model}

The chemistry governing the oxidation of methane is taken into account by the 1-step chemical kinetic mechanism with equivalence ratio correction from Mantel et al. 26]. The Thickened Flame model from Collin et al. [27] is employed to thicken the flame front so that it can be resolved in the LES mesh. A dynamic calculation of the local thickening factor $F$ is employed to correctly compute the flame front in the mesh. The dynamic thickening factor approach from Durand et al. [28] is followed to obtain the local thickening factor $F$ :

$$
F=1+\left(F_{\max }-1\right) \Omega
$$

where $\Omega$ is a sensor detecting the presence of the flame front and $F_{\max }$ is the maximum thickening factor allowed in the computation. This sensor can be expressed as a function of a reaction progress $c$ that is defined as the ratio of mass fraction over stoichiometric mass fraction $c=1-Y_{f} / Y_{f, s t}$ :

$$
\Omega(c)=16(c(1-c))^{2}
$$


$F_{\max }$ is calculated as:

$$
F_{\text {max }}=\frac{N \Delta}{\delta_{l}}
$$

where $\mathrm{N}$ is the total number of nodes to reproduce the gradient $(\mathrm{N}=8$ in our simulation) and $\delta_{l}$ is the laminar flame thickness. The Thickened Flame model also requires a correction of the transport coefficients to recover the Damköhler number of the original flame after the thickening and an efficiency function $\mathrm{E}$ is used [27]. The transport coefficients and source terms in the energy and species mass fraction transport equations Eqs. (3)-(4) are corrected as:

$$
\begin{gathered}
\frac{\partial\left(\overline{\rho c_{p}} \widetilde{T}\right)}{\partial t}+\frac{\partial\left(\overline{\rho c_{p}} \widetilde{u}_{j} \widetilde{T}\right)}{\partial x_{j}}=\frac{D p^{t h}}{D t}+\frac{\partial}{\partial x_{j}}\left(\lambda_{\text {eff }} \frac{\partial \widetilde{T}}{\partial x_{j}}\right)+\left(\bar{\rho} \sum_{k=1}^{N} \bar{c}_{p, k} D_{k, \text { eff }} \frac{\partial \widetilde{Y}_{k}}{\partial x_{j}}\right) \frac{\partial \widetilde{T}}{\partial x_{j}}+ \\
\bar{\tau}_{i j} \frac{\partial \widetilde{u}_{i}}{\partial x_{j}}+\frac{E}{F} \bar{Q}^{c} \\
\frac{\partial\left(\bar{\rho} \widetilde{Y}_{k}\right)}{\partial t}+\frac{\partial\left(\bar{\rho} \widetilde{u}_{j} \widetilde{Y}_{k}\right)}{\partial x_{j}}=\frac{\partial}{\partial x_{j}}\left(\bar{\rho} D_{k, \text { eff }} \frac{\partial \widetilde{Y}_{k}}{\partial x_{j}}\right)+\frac{E}{F} \overline{\dot{\omega}}_{k} \quad k=1, \ldots, N
\end{gathered}
$$

where the effective transport coefficients are given by:

$$
\begin{gathered}
D_{\mathrm{eff}, \mathrm{k}}=\frac{\bar{\mu}}{\bar{\rho} S c_{k}} F+(1-\Omega) \frac{\mu_{t}}{\bar{\rho} S c_{t}} \\
\lambda_{\mathrm{eff}}=\bar{c}_{p}\left(\frac{\bar{\mu}}{P r} F+(1-\Omega) \frac{\mu_{t}}{P r_{t}}\right)
\end{gathered}
$$

being $\bar{\mu}, \bar{\mu}_{t}, S c_{k}, S c_{t}, \operatorname{Pr}$ and $P r_{t}$ the molecular viscosity, turbulent viscosity, Schmidt number of species $k$, turbulent Schmidt number, Prandtl number and turbulent Prandtl number respectively. Both $S c_{t}$ and $P r_{t}$ are set to 0.9 , while the Schmidt number is obtained by assuming a constant Lewis $L e_{k}=S c_{k} / P r_{k}$ and Prandtl numbers for each species and are given in Table 1.

Table 1: Species $\operatorname{Pr}_{k}$ and $\mathrm{Le}_{k}$ numbers.

\begin{tabular}{cccccc}
\hline \hline Species & $\mathrm{CH}_{4}$ & $\mathrm{O}_{2}$ & $\mathrm{~N}_{2}$ & $\mathrm{CO}_{2}$ & $\mathrm{H}_{2} \mathrm{O}$ \\
\hline \hline $\mathrm{Pr}_{k}$ & 0.729 & 0.713 & 0.721 & 0.762 & 0.987 \\
\hline $\mathrm{Le}_{k}$ & 1.06 & 1.1 & 1.0 & 1.4 & 0.9 \\
\hline
\end{tabular}




\subsection{Numerical methods}

The governing equations are solved in the Finite-Element framework using the variational multiscale stabilization approach (VMS) [29] that allows to deal with convection dominated flows and use equal order of interpolation for velocity and pressure. In this work, VMS is not used as a subgrid scale model, but as a numerical stabilization mechanism. Therefore, an explicit subgrid scale model (WALE) is used to close the unresolved terms coming from the filtering operation. A second order Cranck-Nicholson method is used for the time integration.

The discretization of the Navier-Stokes equations yields a coupled algebraic system to be solved at each linearization step within a time loop. The system is split to solve the momentum and continuity equations independently, applying the iterative Orthomin (1) solver for the pressure-Schur complement [30]. At each linearization step, it is necessary to solve the momentum equation twice and the continuity equation once to achieve proper convergence behaviour. The momentum equation is solved using a GMRES solver and the continuity equation is solved using the Deflated Conjugate Gradient method [31. The parallelization of the solver consists of a master-slave strategy and is described in detailed elsewhere 32. The GMRES solver is also employed to solve for the enthalpy and the individual species mass fractions. The Gauss-Seidel iterative method is employed to couple the species mass fraction until the targeted convergence.

\section{Validation exercise}

The validation exercise includes the assessment of chemical kinetics, conjugate heat transfer and fluid flow configuration. The latter will be discussed in the next section.

\subsection{Chemical kinetics assessment}

The chemical scheme proposed by Mantel et al. [26] is used to predict the chemical characteristics of the flame in this study. To evaluate the accuracy, a comparison with the code PREMIX [33] using the GRI Mech 3.0 mechanism [34] is made in a freely propagating 1D laminar premixed flame at equivalence ratio $\phi=0.8$ and $\mathrm{T}=298 \mathrm{~K}$. The comparison of the resolved flame temperature is shown in Fig. 2 in a 1D mesh of 200 elements where the 8 nodes are used to compute the temperature gradient.

The results show acceptable correlation with the reference solution of PREMIX and the flame speed was captured with less than $5 \%$ error. Therefore, the simulations are performed using a minimum of 8 nodes across the flame front to ensure a correct prediction of the gradients. 


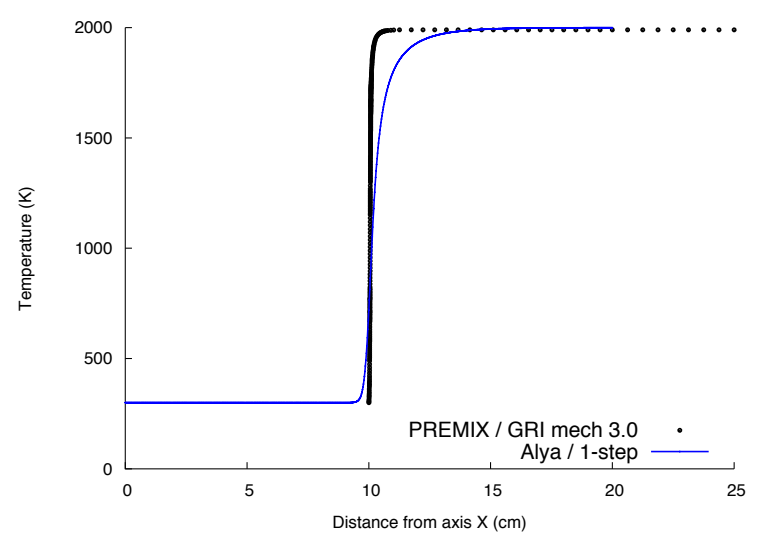

Figure 2: 1D flame temperature using the code Alya with a 1-step model compared to PREMIX.

\subsection{Solid solver assessment}

To validate the conjugate heat transfer solver, a test case of heat conduction between two solids is considered. This test ensures the correct prescription of Dirichlet and Neumann -type boundary conditions among the two domains for CHT problems. Note this test case is set with two solid domains, but is treated with the same approach as the one that is used for CHT problems. The benchmark case with the boundary conditions is sketched in Fig. 3 (a). The problem is set imposing Dirichlet boundary conditions at top and bottom of the domain $\mathrm{T}_{1}$ and $\mathrm{T}_{2}$ with $\mathrm{T}_{1}>\mathrm{T}_{2}$, while adiabatic conditions are prescribed in the $x$ direction. The thermal diffusivities of the two materials satisfy $k_{s, 1}<k_{s, 2}$ and the problem has analytical solution, which is shown in Fig. 3 (b). This simple test case permits an evaluation of the algorithm used to exchange information between subdomains for CHT problems. The results show excellent agreement when compared to the analytical solution validating the coupling approach.

\section{Impinging flame configuration}

\subsection{Physical problem}

The impinging flame configuration considered in this work corresponds to a methane premixed flame with equivalence ratio $\phi=0.8$ at $\mathrm{T}=298 \mathrm{~K}$ impinging on a flat plate. The nozzle-to-plate distance is $\mathrm{H} / \mathrm{D}=2$ and the inlet velocity is set as $30 \mathrm{~m} / \mathrm{s}$. The jet inlet diameter is $\mathrm{D}=0.01 \mathrm{~m}$ and the length of the fluid domain parallel to the plate is 20D. A section of the inlet pipe with length $2 \mathrm{D}$ is also accounted for in the simulation with a cone-shaped bluff body of diameter $\mathrm{d}=0.001$ 


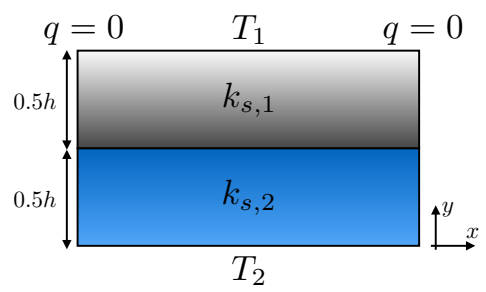

(a)

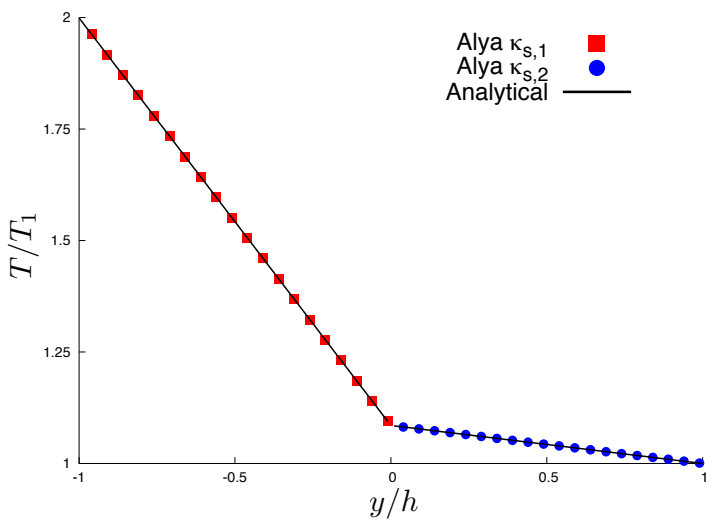

(b)

Figure 3: (a) Conjugate conduction problem definition and (b) numerical results compared to the analytical solution.

and length $l=0.001$ at the center of the inlet tube as those found in practical burners. The solid part is represented by a squared domain of length 20D and thickness 2D. A sketch representing the fluid and solid domains is shown in Fig. 4.

In order to define the thermal properties of the solid domain, the thermal activity ratio $\mathrm{A}$ is used to characterise the thermal fluid-solid interaction. The thermal activity ratio is defined as:

$$
A=\sqrt{\frac{\left(\rho c_{p} k\right)_{f}}{\left(\rho c_{p} k\right)_{s}}}
$$

where the subscript $f$ refers to fluid and $s$ to solid. This ratio characterises the thermal behaviour of a solid interface and leads to two opposite situations. When $\mathrm{A} \rightarrow 0$, the interface can be considered isothermal and no fluctuations are expected, whereas when $\mathrm{A} \rightarrow \infty$, maximum level of fluctuations are expected at the interface and a constant heat flux is found [35]. In addition, taking into account the thermal activity ratio and the synchronization in physical time between fluid and solid, the material conductivity of the solid was enhanced by a factor of 100, while the rest of properties were considered as that of $0.5 \% \mathrm{C}$ steel. It means that the solid response to changes in the fluid domain is enhanced allowing to obtain faster variations in temperature across the solid and better visualize the cooling effects on the impinging plate at the temporal scales given by the fluid. In this case, the thermal activity ratio is $\mathrm{A} \approx 7.0 \mathrm{E}-05$, which is different from the isothermal case $(\mathrm{A}=0)$.

\subsection{Numerical setup and boundary conditions}

The computational domain with the corresponding boundary conditions is sketch in Fig. 4. The velocity profile at the inlet is imposed with a top hat profile and the 
turbulence is generated in the wake of the bluff body. The inflow condition for the temperature and species mass fractions is prescribed with $\mathrm{T}=298 \mathrm{~K}$ and mass fractions corresponding to $\phi=0.8$. All walls are set no-slip. The domain has been extended in the radial direction coarsening the grid to allow for the formation of a sponge layer and outflow conditions are applied for all variables at the outlet. All solid walls are assumed adiabatic except the impinging wall, at which different thermal conditions are imposed.

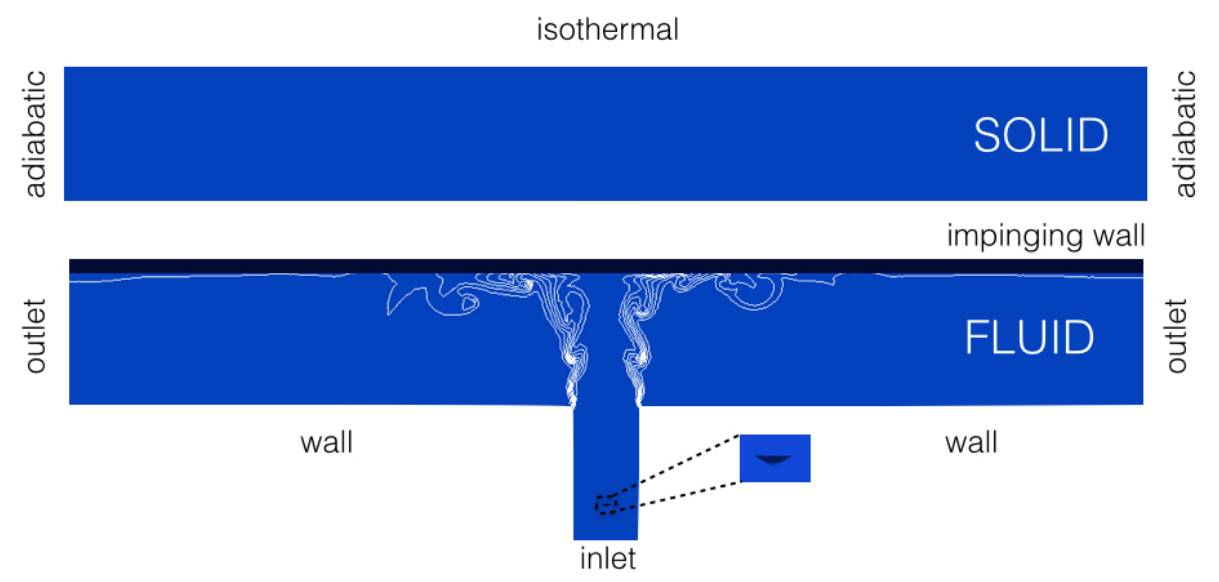

Figure 4: Computational domain and boundary conditions.

Five different heat transfer conditions at the impinging wall are examined in this study. Four simplifications that avoid the need of modelling the solid and the entire coupled problem are compared to the CHT case, where fluid and solid are solved simultaneously. The first case assumes an adiabatic wall, the second case corresponds to an isothermal wall at $\mathrm{T}_{w}=900 \mathrm{~K}$ and the third imposes a constant heat flux. The fourth case uses a Robin-type boundary condition of the form $q=h\left(T-T_{w}\right)$ for the heat flux at the plate. In this approach, the $T_{w}$ represents the wall temperature set to $900 \mathrm{~K}$ and $h$ is the effective heat transfer coefficient. The fifth case computes the heat flux using the conjugate heat transfer (CHT) approach and imposes skin temperatures on the fluid side, and heat fluxes on the solid side [36].

To determine the heat flux $q$ and heat transfer coefficient $h$ required to set Cases 3 and 4 , the fields of the isothermal wall at $\mathrm{T}_{w}=900 \mathrm{~K}$ are used. From the precursor simulation, one can obtain the averaged heat flux over the impinging plate integrating the mean heat flux over the plate area $\partial S$ as:

$$
q=\int_{\partial S}\langle q\rangle d S=-\int_{\partial S}\langle k \nabla T\rangle d S
$$

This heat flux is used to set Case 3 and is used to evaluate the effective heat transfer coefficient $h$ required for Case 4 . The value of $h$ is obtained so that a given heat 
flux at the impinging wall is obtained. Therefore, the value of $h$ is linearly increased until matching the same heat flux as the previous cases. Note, the fluid temperature at the wall in this case is higher than that of the isothermal case $\left(\mathrm{T}=\mathrm{T}_{w}\right)$, since this relation is satisfied:

$$
T=T_{w}+\frac{q}{h}
$$

To set the CHT case, the isothermal temperature on the upper side of the solid domain must be prescribed. This temperature is set as $\mathrm{T}_{s}=800 \mathrm{~K}$, so this case represents a thermal case in between the isothermal and the case represented by a constant heat transfer coefficient (Case 4). As the thermal activity ratio in this case is small, the solid domain will behave as a non-isothermal solid allowing variations of temperature and fluctuations at the plate. Cases 3 and 4 should be considered as a simplification of the CHT case, in which a similar heat flux is predicted but the response of the fluid due to the numerical condition at the plate differs. Case 4 should be considered as an approximation of the CHT case, for which a deficit in the effective heat transfer coefficient is assumed. A summary of the computational cases is given in Table 2 .

Table 2: Computational cases.

\begin{tabular}{cc}
\hline \hline Cases & Thermal condition \\
\hline \hline Case 1 & Adiabatic $(\mathrm{q}=0)$ \\
\hline Case 2 & Isothermal $\left(\mathrm{T}_{w}=900 \mathrm{~K}\right)$ \\
\hline Case 3 & Isoflux $(\mathrm{q}=500 \mathrm{~W})$ \\
\hline Case 4 & $\mathrm{~h}\left(\mathrm{~T}-\mathrm{T}_{w}\right)$ \\
\hline Case 5 & Conjugate $(\mathrm{CHT})$ \\
\hline
\end{tabular}

The boundary conditions specified at the impinging wall in the fluid domain (see Fig. (4) for each case are summarised here:

\section{Adiabatic}

$$
-k_{f} \frac{\partial T}{\partial \vec{n}}=0
$$

where $\vec{n}$ is the normal pointing outwards of the wall.

Neumann-type (isoflux)

$$
-k_{f} \frac{\partial T}{\partial \vec{n}}=q
$$

Robin-type

$$
-k_{f} \frac{\partial T}{\partial \vec{n}}-h\left(T-T_{w}\right)=0
$$


Conjugate heat transfer (CHT)

The boundary condition at the interface between solid and fluid $\partial S$ is obtained after solving the coupled thermal problem. The solution is accomplished in several steps [37]:

- Solve heat conduction equation in the solid domain $\Omega_{s}$ knowing the interface heat flux $q^{n}$ given by the fluid domain.

- Impose skin temperature on $\partial S$ of the fluid domain using a relaxation factor $\alpha$ :

$$
T_{f}^{n}=(1-\alpha) T^{n}+\alpha T^{n+1} \quad \text { on } \partial S \text { where } \alpha \in[0,1]
$$

- Solve transport equations in the fluid domain $\Omega_{f}$ knowing the skin temperature at the fluid/solid interface $\partial S$.

- Obtain updated interface heat flux $q^{n+1}$ and impose Neumann condition on the solid interface $\partial S$ using a relaxation factor $\beta$ :

$$
q^{n+1}=(1-\beta) q^{n}+\beta q^{n+1} \quad \text { on } \partial S \text { where } \beta \in[0,1]
$$

In this work, no outer iterations are used to converge the coupled linear problem, and the relaxation factors $\alpha$ and $\beta$ are constant and set to 1 .

\subsection{Resolution assessment}

The resolution in the fluid domain was examined by testing three computational meshes of 3.6, 4.1 and 5.2 million elements respectively in cold flow. The results of the three cases were compared for the mean and fluctuations resulting in similar trends for the mean values, but not for the fluctuating quantities. The results are presented in the next section. The maximum normalized wall distance $\mathrm{y}^{+}$computed using the half element size at the impinging wall was 21, 12 and 5 respectively. As the Reynolds number diminishes in reacting flows due to the increase of viscosity, an increase of the boundary layer thickness is expected for the reacting case. As it is intended to have a $\mathrm{y}^{+}<10$, the mesh with 5.2 million elements was selected to run all the simulations. The resolution of the flame front was examined by the local thickening factor obtained in the simulations. A maximum local thickening factor of 12 was observed in all four cases in regions away from the wall with the finest grid as recommended by Collin et al. [27]. The mesh was designed to allow a local thickening factor of one near the wall avoiding artificial variations of the heat transfer coefficients by the thickening effects of the flame. 


\section{Results}

In this section, the outcomes of the study are presented. The work is focused on providing further understanding on cooling and heat transfer effects in reactive systems. Two different subsections corresponding to aspects of engineering interest of impinging flames are examined here along with a first section for validation of the cold flow configuration. The time integration of the instantaneous fields is obtained after time-averaging the data for two flow through calculations using the averaged radial velocity. The time integration is considered after the fields are statistically independent and no effect of the initial conditions exist.

\subsection{Cold flow validation}

In this subsection, the LES results are compared against the experimental data of Tummers et al. 38 for mean and fluctuating quantities in a cold configuration and the results are presented in Figs. 5 to 9 respectively. The LES simulation was conducted using a turbulent inlet profile from a precursor simulation instead of using a synthetic eddy method [39]. To obtain the turbulent inlet boundary condition, a pipe flow simulation was performed matching the mass flow rate of the experiments. The pipe flow was run until a turbulent developed flow field was achieved. The velocity components at the inlet plane were stored for each time-step creating a turbulent inlet database that was used as an inlet boundary condition for the impinging jet simulation.

Due to the short distance between the jet and the wall, the velocity profile has a major influence on the fluctuating quantities near the wall. In preliminary tests, using simpler inlet boundary conditions, the mean values were well captured, but the use of a well developed turbulent inlet was mandatory in order to predict the level of fluctuations that match the experimental data. A strong influence of the inlet profile on the fluctuating quantities was observed in this case. The results indicate an acceptable level of correlation with the experiments for the mean and turbulent quantities despite the relatively low resolution of the grid compared to the ones used in the literature [40, 41, 42]. The prediction of the mean axial and radial velocities are in good agreement with the experimental data. The maximum of the radial velocity is very well captured and the discrepancy occurs far from the wall where the boundary condition of the coflow was set with zero radial velocity. In the case of the fluctuations, the trend is maintained and the level of fluctuations matches well the experimental data. Note, the computed Reynolds shear stress in Fig. 9 does not account for the contribution of the subgrid scale part, so that the comparison would be expected to improve when including this contribution. The accuracy is expected to improve for the reacting case, since the Reynolds number is substantially reduced by the increase of viscosity with temperature. 

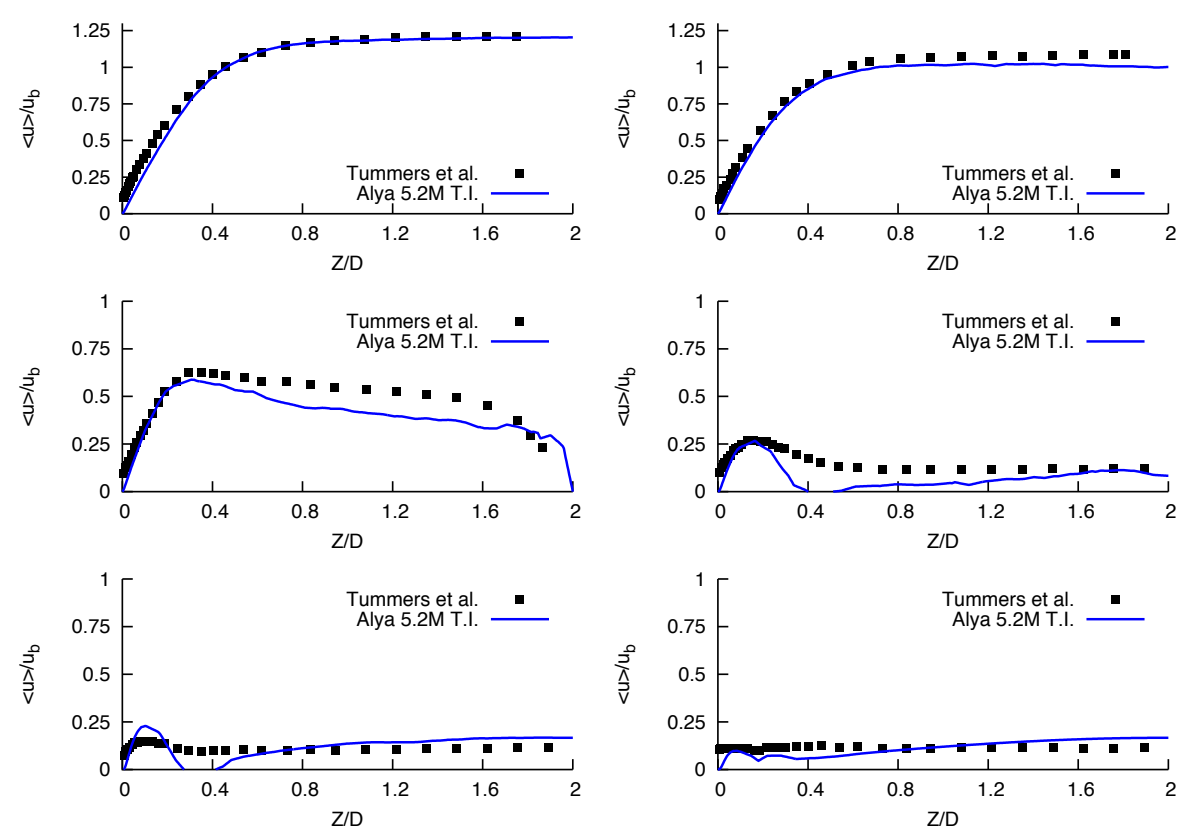

Figure 5: Mean streamwise velocity of the cold flow at different radial positions $r / \mathrm{D}=0.0, r / \mathrm{D}=0.25, r / \mathrm{D}=0.5, r / \mathrm{D}=0.75, r / \mathrm{D}=1.0, r / \mathrm{D}=1.5$.
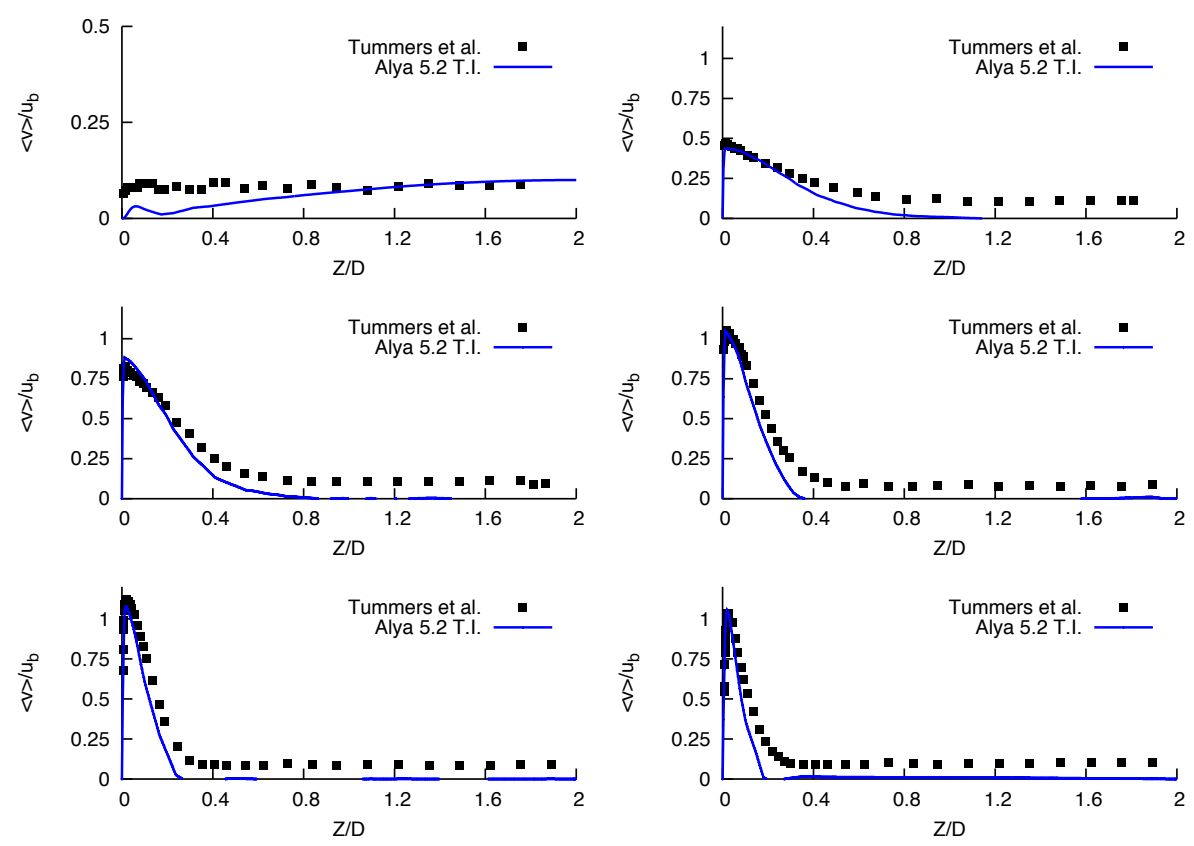

Figure 6: Mean radial velocity of the cold flow at different radial positions $r / D=0.0$, $r / \mathrm{D}=0.25, r / \mathrm{D}=0.5, r / \mathrm{D}=0.75, r / \mathrm{D}=1.0, r / \mathrm{D}=1.5$. 

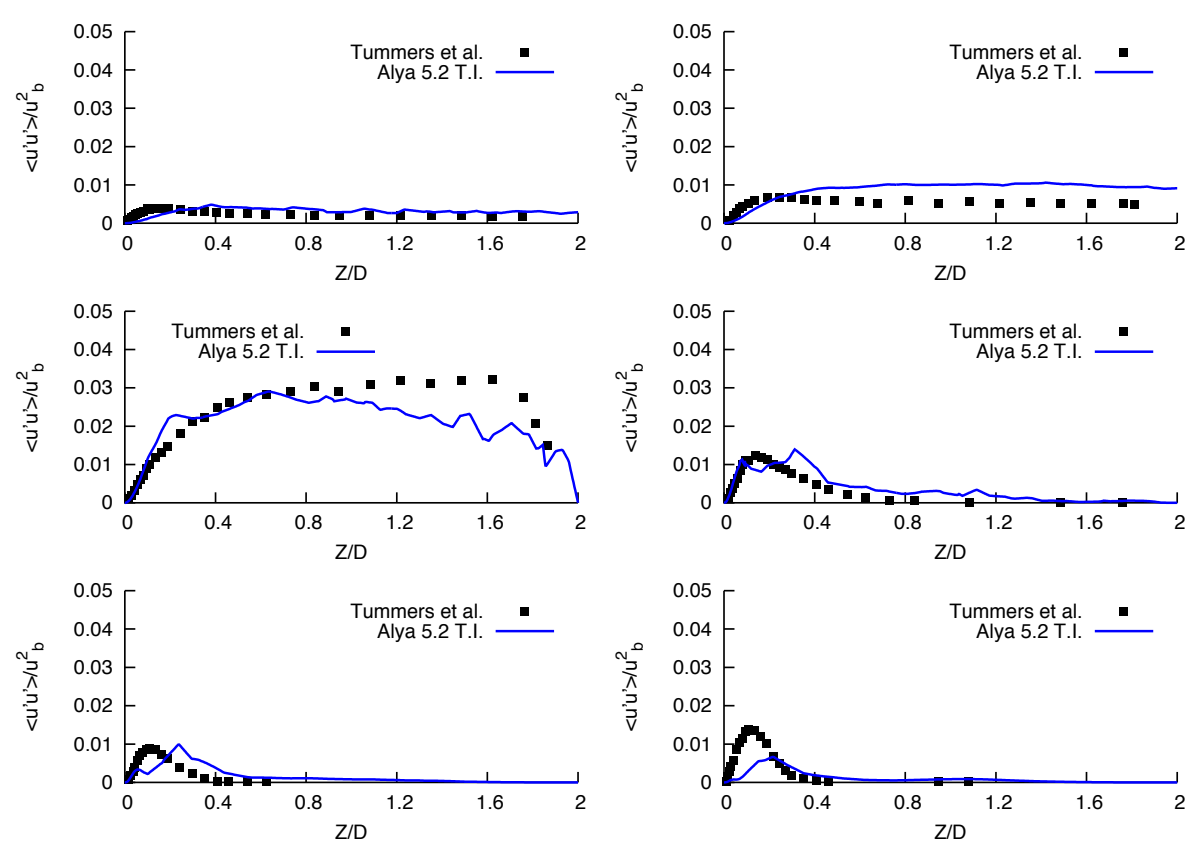

Figure 7: Reynolds normal stress of the streamwise velocity of the cold flow at different radial positions $r / \mathrm{D}=0.0, r / \mathrm{D}=0.25, r / \mathrm{D}=0.5, r / \mathrm{D}=0.75, r / \mathrm{D}=1.0, r / \mathrm{D}=1.5$.

\subsection{Heat transfer}

As the thermal condition at the plate is varied among the cases, the heat transfer is responsible of the changes in the flow field. Figure 10 plots the time evolution of the heat flux and temperature integrated over the plate, which shows the global response of the impinging wall to flame heating.

The temporal trends indicate that all the cases, except the adiabatic case, dissipate approximately the same amount of heat after initialization. However, as the mechanism of heat transfer changes from case to case, the averaged temperature over the plate shows some differences. In particular, the CHT (very low A) and adiabatic $(\mathrm{A}=\infty)$ cases achieve the lower and upper limits of wall temperature among the cases. The wall temperature for the adiabatic case reaches values around the equilibrium temperature $\left(\mathrm{T}_{\mathrm{ad}}=1996.5 \mathrm{~K}\right)$ as no heat losses are taken into account, while the lower bound is given by the CHT case, in which the skin temperature is influenced by the outer wall temperature $\mathrm{T}_{s}$. The other cases are found in between and have a similar response from the perspective of global heat exchange.

The spatial distribution of temperature gradients at the impinging plate provides valuable information about the heat transfer performance of the cases. This is shown in Fig. 11 as contour plots of temperature gradients at the wall (left) and probability distribution of the gradients at that plane (right). A distribution of the temperature 

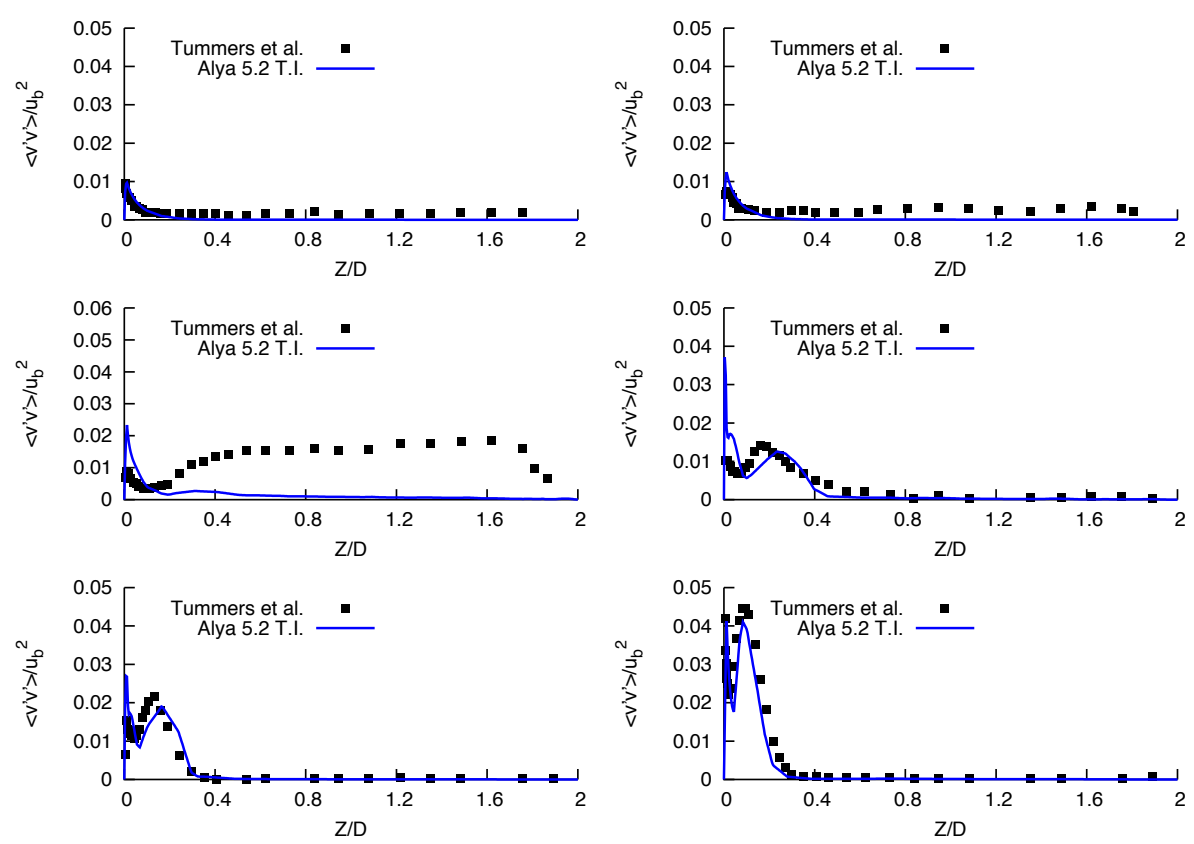

Figure 8: Reynolds normal stress of the radial velocity of the cold flow at different radial positions $r / \mathrm{D}=0.0, r / \mathrm{D}=0.25, r / \mathrm{D}=0.5, r / \mathrm{D}=0.75, r / \mathrm{D}=1.0, r / \mathrm{D}=1.5$.

gradient over the normalized surface area is also shown on the right-plot of Fig. 11. It associates the magnitude of the gradients to the portion of area on which they are distributed relating the sensibility of the boundary respect to changes in flame temperature. For adiabatic conditions, a zero temperature gradient is observed and has been omitted. The right-plot shows that temperature gradients are quite large and occur at particular locations of the wall. The CHT case undergoes the highest temperature gradient, while the case with constant heat flux has the minimum. The other two cases can be found in between. It is also noticeable that the CHT and the isothermal case show narrow distributions indicating that gradients are rather large over particular sections of the plate. The use of a convective wall function of the form $\mathrm{h}\left(\mathrm{T}-\mathrm{T}_{w}\right)$ widens the gradient distribution and enhances the heat exchange when compared to the isoflux case. In general, it is observed that boundary conditions based on flux prescriptions tend to widen the pdf distribution, lowering the peak of the curve and inducing more uniform temperature gradients over the solid plate. Dirichlet-type conditions favour localized temperature gradients that can be larger than Neumann-type boundary conditions at particular locations.

The use of different thermal boundary conditions lead to different spatial distribution of temperature and fluctuations in the near-wall region. Mean temperature $\langle T\rangle$ and temperature fluctuations $\left\langle T^{\prime 2}\right\rangle$ profiles at different radial locations are shown 

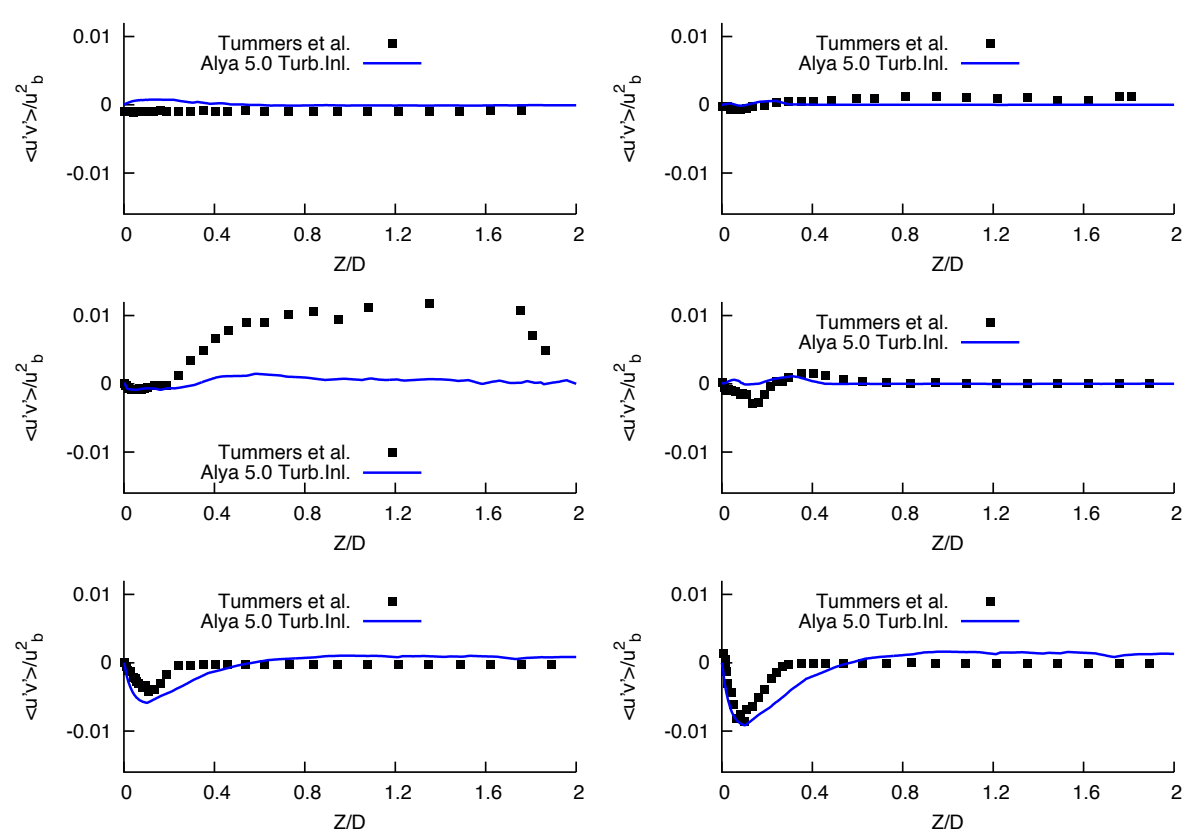

Figure 9: Reynolds shear stress of the cold flow at different radial positions $r / D=0.0$, $r / \mathrm{D}=0.25, r / \mathrm{D}=0.5, r / \mathrm{D}=0.75, r / \mathrm{D}=1.0, r / \mathrm{D}=1.5$.

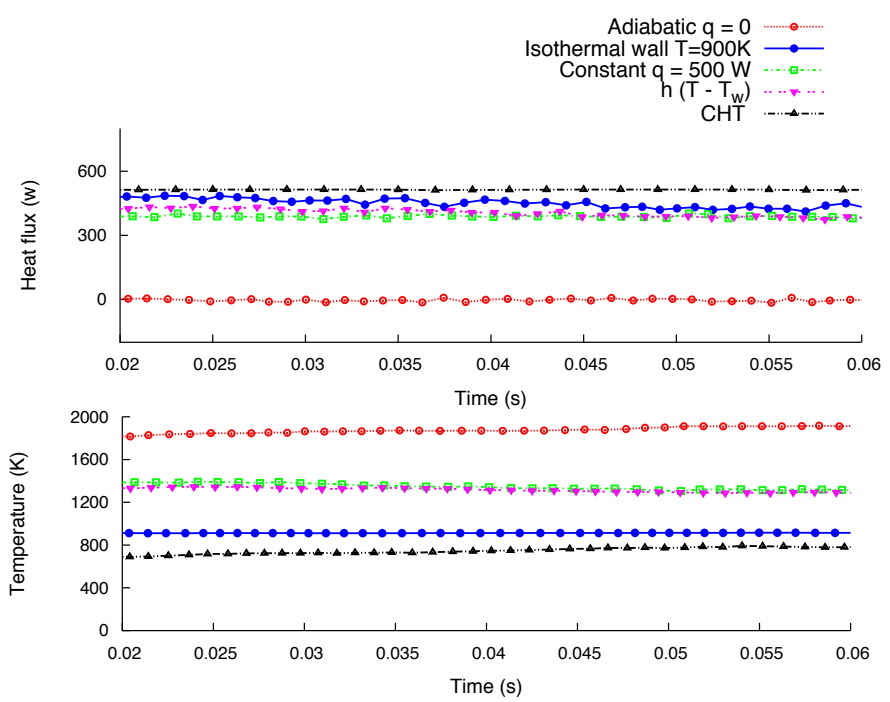

Figure 10: Time evolution of the wall heat flux and temperature after initialization. 


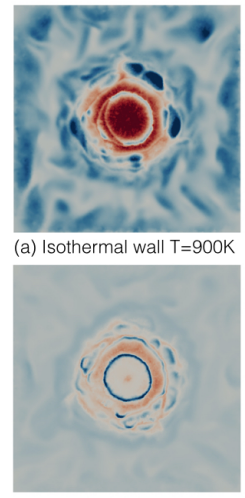

(c) $h\left(T-T_{w}\right)$
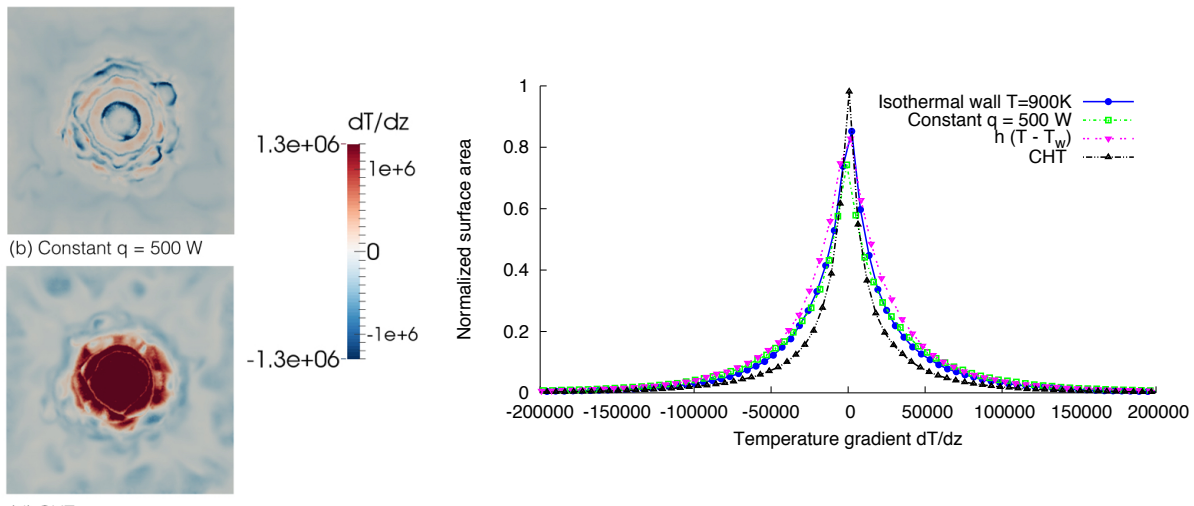

Figure 11: Instantaneous temperature gradients (left) and distribution of temperature gradients (right) at the impinging plate at time $\mathrm{t}=0.045 \mathrm{~s}$.

in Figs. 12 and 13 from the stagnation point outwards (a-d). The values of temperature are normalized with the adiabatic flame temperature $\mathrm{T}_{\mathrm{ad}}$, while the vertical direction $z$ is normalized with the inlet diameter $\mathrm{D}$ and oriented to have $z=1$ at the wall. The plot shows the temperature distribution of the combustion products

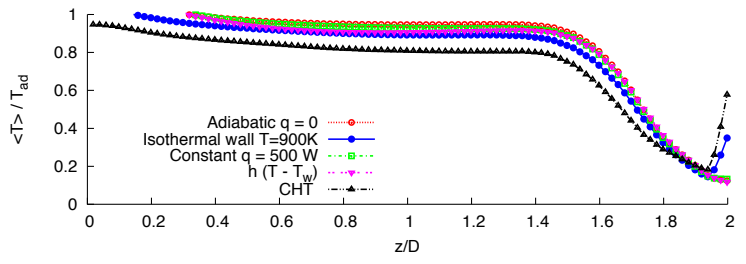

(a)

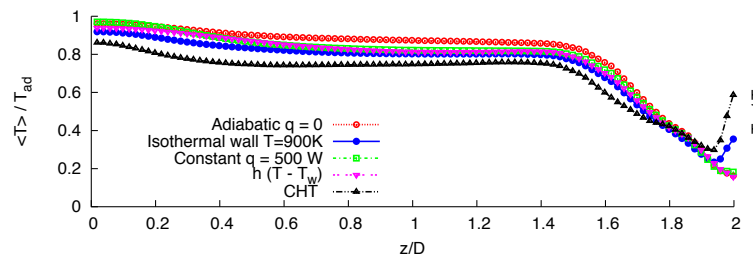

(c)

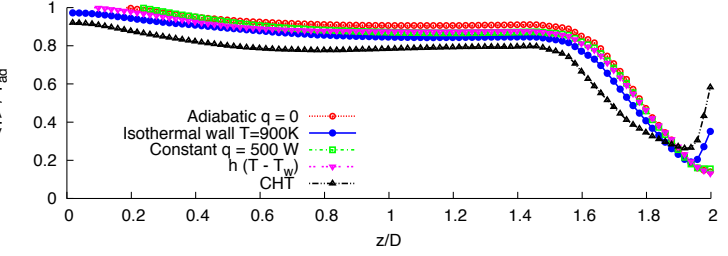

(b)

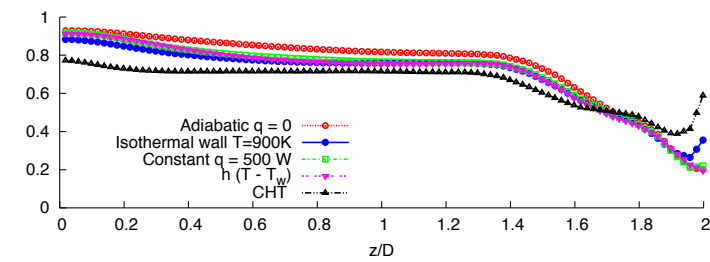

(d)

Figure 12: Mean temperature at different radial locations (a) $r / \mathrm{D}=1.0$, (b) $r / \mathrm{D}=2.0$, (c) $r / \mathrm{D}=2.5$ and $(\mathrm{d}) r / \mathrm{D}=3.0$.

in the domain at different radial locations $r / D$. The cases show a rather constant temperature distribution in the domain with a temperature variation as the flow approaches the deflection zone. The main temperature gradients occur in the nearwall region, in which the flow has to satisfy the boundary condition. The cases with constant heat flux and convective function show a similar behaviour, since the 
gradient is smooth for the first, and the effective heat transfer coefficient $h$ is small to enforce strong temperature gradients. In particular, the adiabatic case shows no gradient at the wall, since this is imposed by the Neumann condition. The isothermal and CHT cases must satisfy the Dirichlet condition at the wall, and substantial reduction of the local value of temperature is found as the flow approaches the wall. In the particular case of the CHT, the temperature is imposed at the interface from the conjugate problem and the value depends on properties from both fluid and solid domains. As the thermal activity ratio is small, though different from zero, a temperature gradient is formed allowing a spatial distribution of temperature changing in time. The temperature profiles are different among the cases, despite a similar heat flux is dissipated. The other cases also show small temperature variations at different locations. The minimum temperatures found at most locations correspond to the CHT case, in which the heat transfer condition imposes the largest heat flux at the wall. The cases with constant heat flux and convective wall function behave alike for most locations and some disparities can be found in the near-wall region due to the different thermal condition. In particular, the temperature at the wall is slightly lower for the convective function condition. The differences between the cases close to the lower wall $(z / \mathrm{D}<1.4)$ are associated to the formation of large vortices that transport the hot products of combustion down to the lower wall reducing the temperature gradient in the $z / \mathrm{D}$ direction.

The profiles of temperature fluctuations given in Fig. 13 also confirm the influence of the thermal condition in the heat transfer performance of the investigated flames. Despite the magnitude of the fluctuations is rather similar for all the cases, the difference among all the cases become more evident in the near-wall region. In this region, the level of fluctuations reduces for all the cases. While the adiabatic case allows the fluctuations to propagate at the wall, the isothermal case eliminates them completely as the flow comes closer to the plate. The rest of cases can be found in between. This result indicates that traditional or advanced thermal conditions affects substantially the dynamics of the flow in the near-wall region, and also the dumping of the turbulent fluctuations. The uncertainty associated to the thermal wall conditions can be reduced by the use of a conjugate approach, which accounts for the properties and materials of the solid structures.

\subsection{Flow dynamics}

The main flow characteristics of an impinging flame can be seen by the instantaneous temperature plots shown in Fig. 14 for the CHT case. It shows the main regions of an impinging flame: the free jet region, the deflection zone, stagnation point and the development of the wall jet. It shows the existence of a thermal boundary layer where the temperature reduces substantially towards the wall. The temperature at the interface corresponds to the conjugate problem, for which an enhancement of the thermal conductivity is given to the solid domain. Using these 


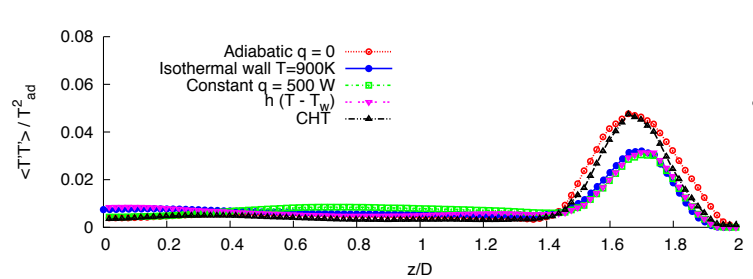

(a)

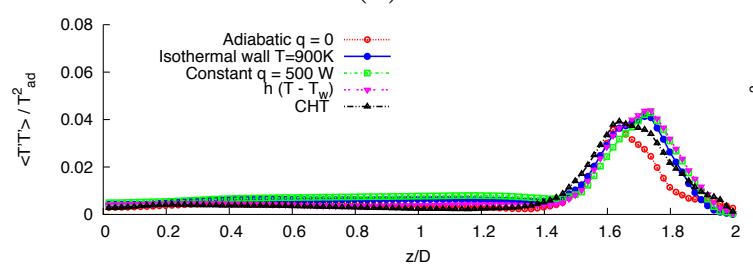

(c)

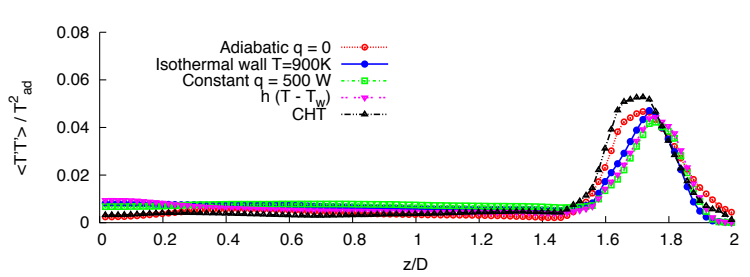

(b)

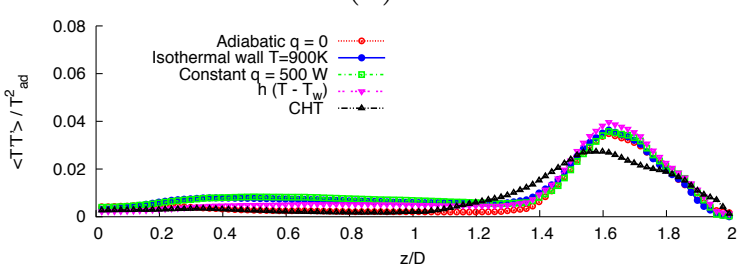

(d)

Figure 13: Temperature fluctuations at different radial locations (a) $r / \mathrm{D}=1.0$, (b) $r / \mathrm{D}=2.0,(\mathrm{c}) r / \mathrm{D}=2.5$ and $(\mathrm{d}) r / \mathrm{D}=3.0$.

properties, the solid domain is able to interact with the temperature fluctuations of the fluid showing a rapid response. The dynamics of such flames can further be described by Q-vorticity contours as seen in Fig. 15. The creation and development of large vortex rings can easily be distinguished in Fig. 15. Small-scale vortices are developed at the shear layer by Kevin-Helmholtz instabilities and are convected radially by the flow in the deflection zone forming large-scale vortex rings, where flow transfers axial to radial momentum. These vortices interact with small-scale structures in the near-wall region affecting the local dynamics of the flow.

The heat transfer condition influences the formation, convection and size of the vortex rings as well as the dynamics of the flame in the deflection region. This can be seen in Fig. 16 by vertical profiles of time-averaged heat release at several radial locations. In general, most cases show similar trends near the lower wall, but some discrepancies appear in the near-wall region of the impinging plate. The differences found at the lower wall can be attributed to the different dynamics of the flames. This is more noticeable for the CHT case. In the free jet region, the heat release distributions is rather similar for all the cases, and the fundamental differences start appearing in as the radius $(\mathrm{r} / \mathrm{D})$ increases. The CHT case delivers the lowest heat release rate, since the combustion process is highly influenced by the strong heat losses to the walls and the temperature near the wall reduces (see also Fig. 12). The same effect can also be seen in the isothermal case, for which the heat release rates are also lower than those of isoflux conditions. In the opposite side, the adiabatic case shows the largest heat release rates, while the cases with Robin-type conditions are in-between. The thermal condition imposed on the solid plate causes different patterns of heat release rate, which in turn affect the dynamics of the flames. 


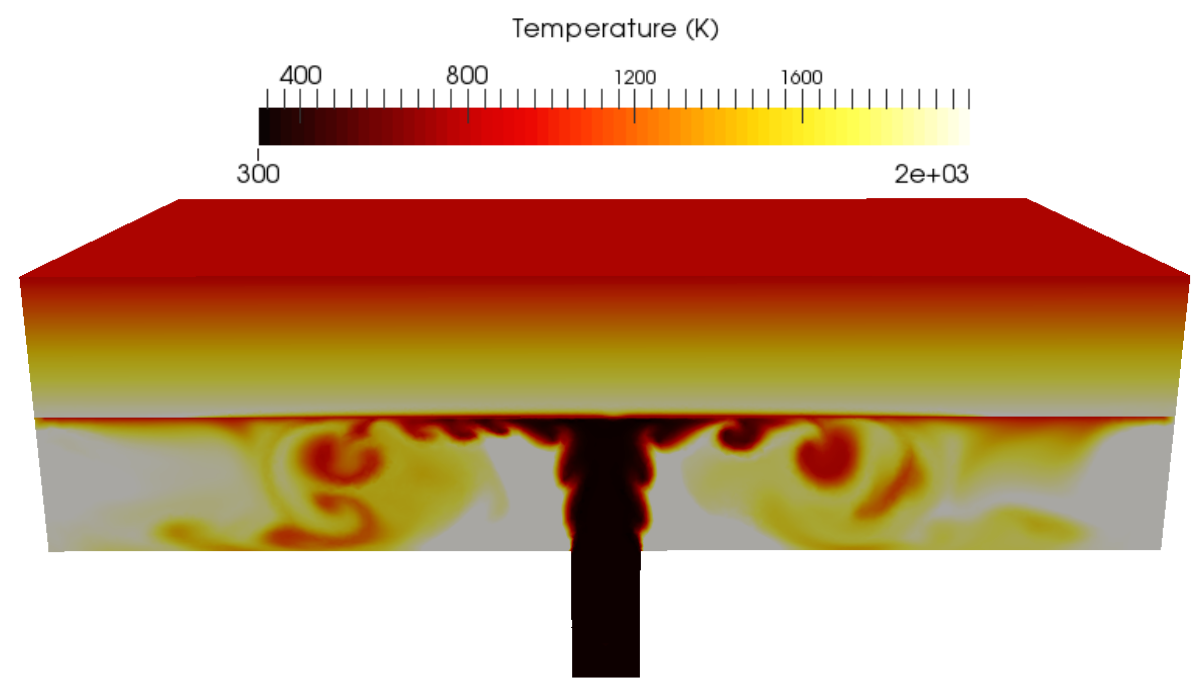

Figure 14: Temperature contour plot for the CHT case for both fluid and solid domains at time instant $\mathrm{t}=0.050 \mathrm{~s}$.
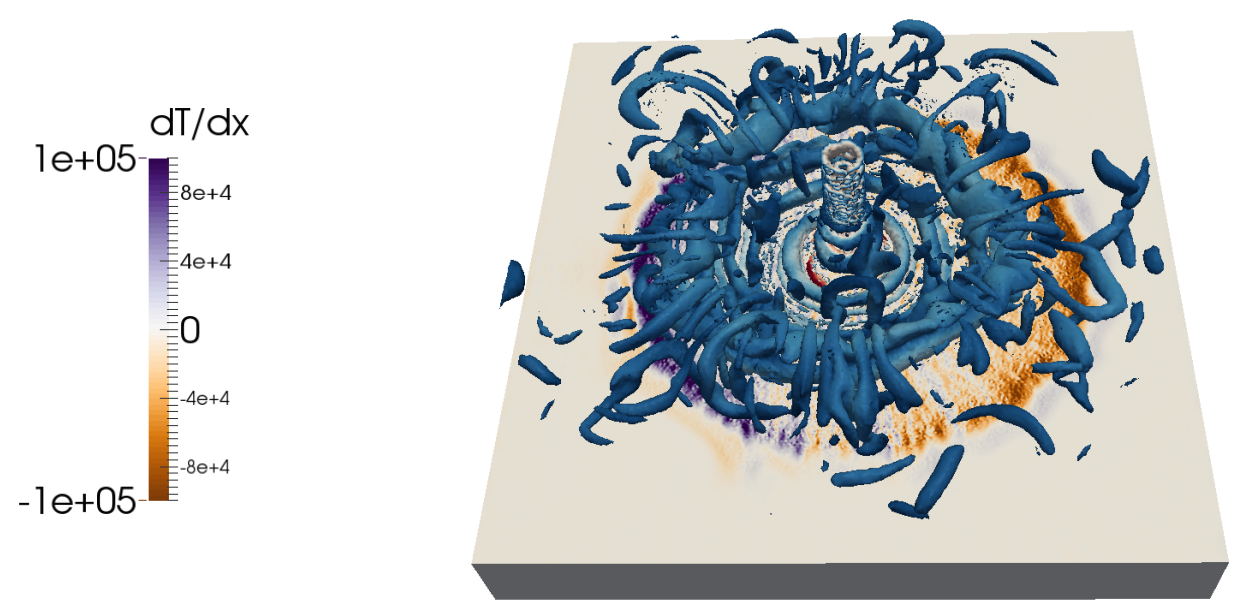

Figure 15: Q-vorticity plot of the CHT case on top of the solid domain coloured by temperature gradients at time instant $\mathrm{t}=0.050 \mathrm{~s}$. 


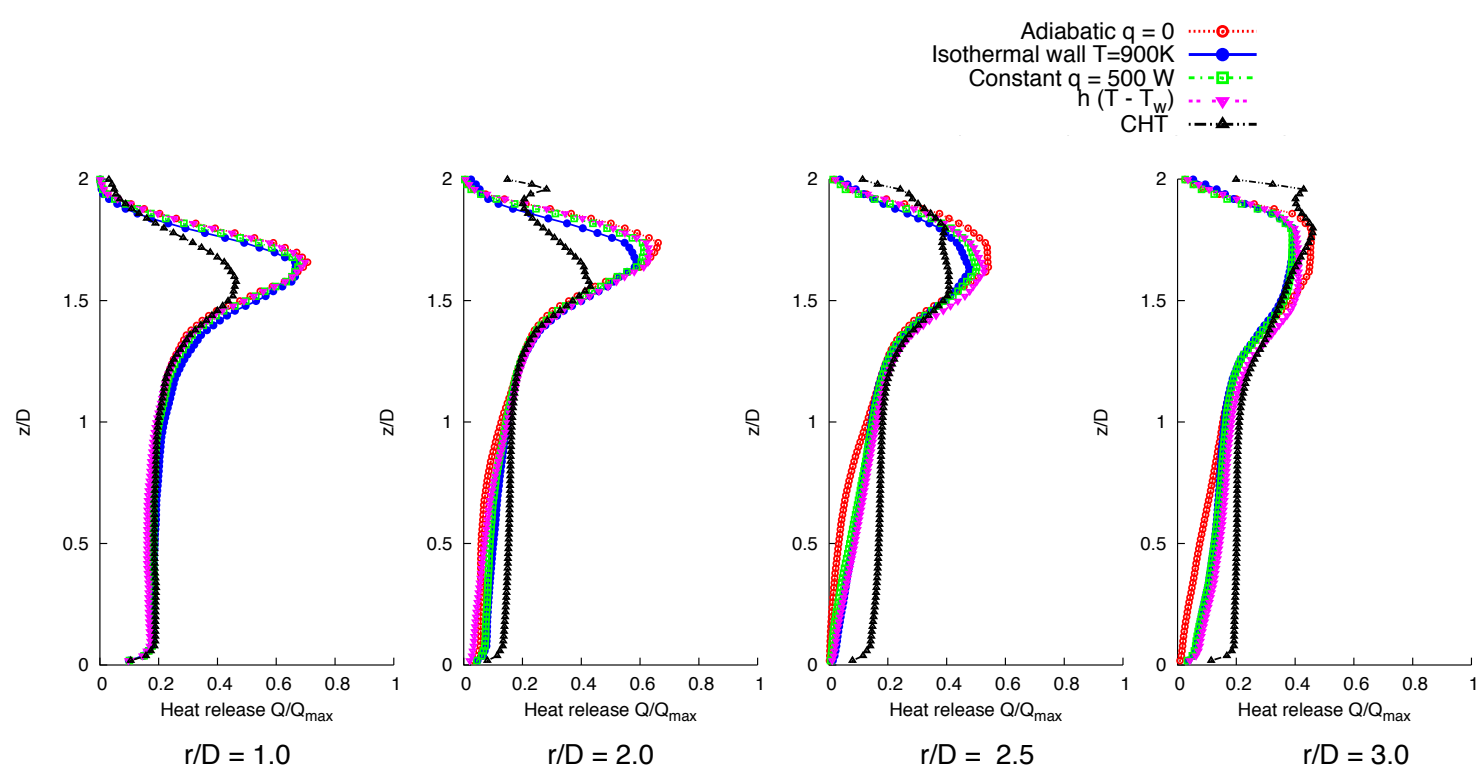

Figure 16: Profiles of normalized mean heat release at different radial locations $r / \mathrm{D}$.

An important phenomena in impinging jets is the formation of a jet parallel to the plate. To examine the creation of a boundary layer and the formation of a wall jet, mean radial velocity $\langle v\rangle$ and velocity fluctuations $\left\langle v^{\prime 2}\right\rangle$ profiles at different radial locations $r / \mathrm{H}$ are shown in Figs. 18 and 19 . As the global heat flux is rather similar between the cases, no substantial differences would be expected in the flow field. Despite the velocity distribution is qualitatively similar among the cases, a different acceleration of the flow occurs for the cases with higher heat losses. For the cases with larger temperature gradients (isothermal and CHT), an enlargement of the thickness of the wall jet occurs due to effects of turbulent mixing and convection in the near-wall region. This effect reduces the maximum velocity of the jet and affects the shear stress in the radial direction. This is shown in Fig. 17, where the mean shear stress is plotted. The results of the wall shear stress are in the same range as the values from Tummers et al. [38], although the latter correspond to non-reacting flow. The turbulent fluctuations in the radial directions confirm the same trend. High level of fluctuations are mainly generated in the near-wall region, showing two peaks along the jet. The level of the fluctuations is similar in the two regions, but as the jet develops outwards radially, the level of fluctuations increases leading to a maximum at around $r / \mathrm{D}=1.8$. From $r / \mathrm{D}=1.0$ to $r / \mathrm{D}=2.0$, the turbulence intensity for the CHT is higher than the rest of the cases, and induces more mixing and turbulent diffusion that eventually reduces the axial momentum of the wall jet.

The same boundary conditions for the velocity are imposed for all the cases and 


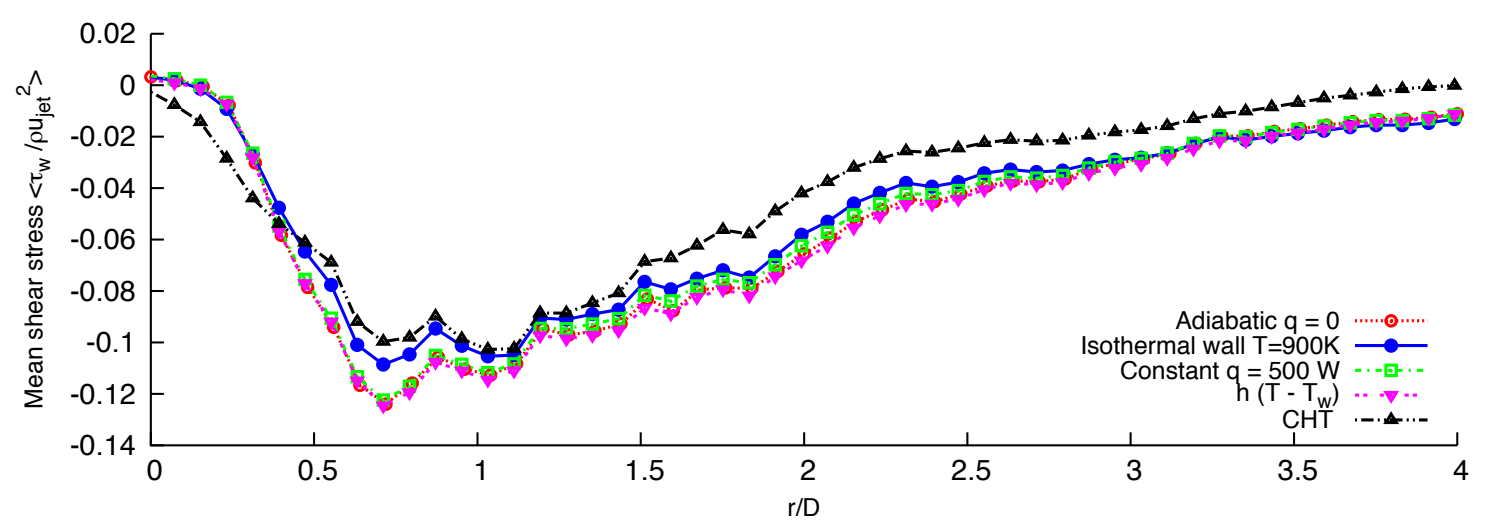

Figure 17: Mean wall shear stress along the radial direction.

the dissimilar trends are associated to the different numerical thermal condition. Compared to the plots of temperature at the same location (see Figs 12 and 13), the thermal condition has a minor effect on the formation of the wall jet, although it induces some local effects as seen in Figs. 18 and 19.

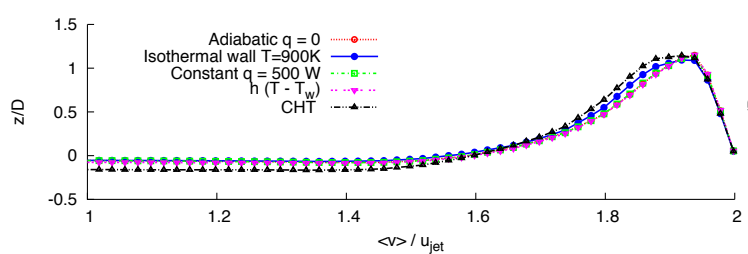

(a)

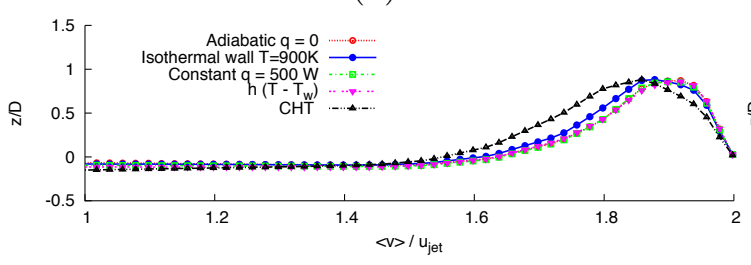

(c)

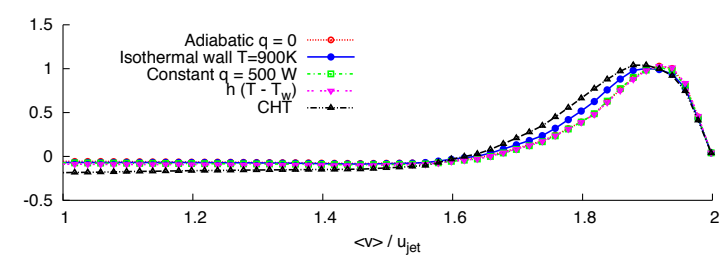

(b)

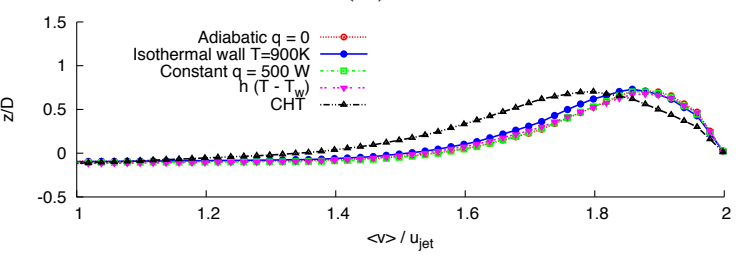

(d)

Figure 18: Mean velocity at different radial directions (a) $r / \mathrm{D}=1.0$, (b) $r / \mathrm{D}=2.0$, (c) $r / \mathrm{D}=2.5$ and $(\mathrm{d}) r / \mathrm{D}=3.0$.

\section{Conclusions}

This paper presents a numerical study based on large-eddy simulation of a premixed impinging flame exiting at $30 \mathrm{~m} / \mathrm{s}$ and $\mathrm{T}=298 \mathrm{~K}$ for a configuration of nozzle-to-plate distance over diameter of $\mathrm{H} / \mathrm{D}=2$. The study is motivated by the 


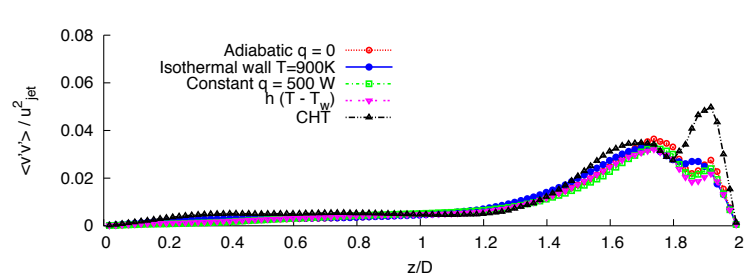

(a)

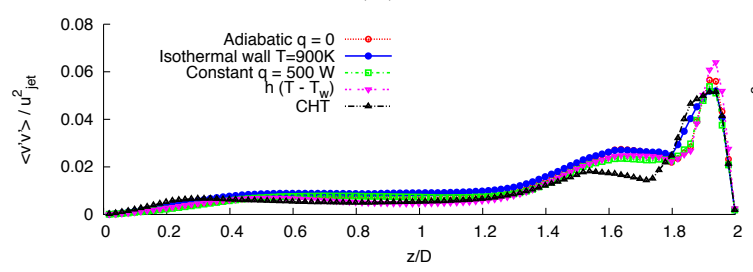

(c)

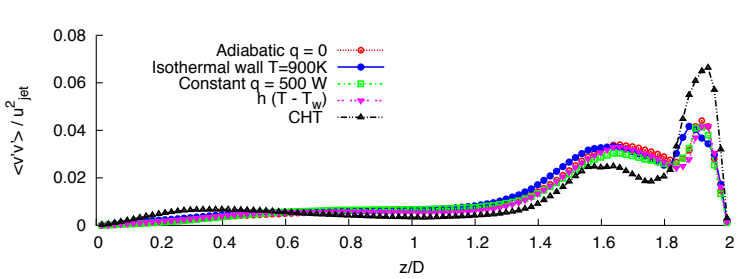

(b)

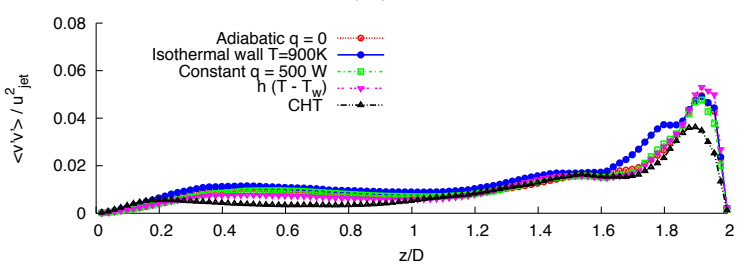

(d)

Figure 19: Root-mean square of velocity fluctuations at radial directions (a) $r / \mathrm{D}=1.0,(\mathrm{~b}) r / \mathrm{D}=2.0$, (c) $r / \mathrm{D}=2.5$ and (d) $r / \mathrm{D}=3.0$.

uncertainty associated to the prescription of thermal boundary conditions in confined geometries of reactive systems. In general, Dirichlet, Neumann or Robin-type boundary conditions are employed, but no further analysis of their effects on the flow field are provided. This work is focused on the analysis and description of the effects that thermal boundary conditions have on the heat transfer performance and flame dynamics of an impinging flame. The cases are carefully selected so that a similar net heat flux is dissipated through the plate, but with different effective heat transfer conditions. These cases are also compared to the case in which fluid and solid domains are solved simultaneously using a conjugate heat transfer approach in a solid with enhanced conductive properties. For this conjugate case, the use of a coupling algorithm for parallel communication to exchange data between different domains is described and validated for thermal problems. A validation of the chemistry and the cold flow are also provided before conducting the reacting simulations. The results reveal substantial differences when the boundary conditions are varied at the impinging wall. The differences among the cases not only affect the mean temperature and gradients in the near-wall region, but also the temperature fluctuations and dynamics of the flow. The distribution of gradients over the plate indicates that Neumann-type boundary conditions induce a more uniform temperature gradients, while Dirichlet-type conditions tend to create local regions with high temperature gradients. In particular, temperature variations, gradients and fluctuations in the near-wall region also require information of the solid domain, and that can only achieved by the use of a conjugate heat transfer approach. Different boundary conditions underpredict/overpredict the fluctuations and might lead to wrong predictions of the thermal behaviour of the flow close to the wall. In the 
particular case of a solid wall with high conductive properties, the temporal scales in the solid are of the same order as those of the fluid, enhancing the response of the solid to changes in the fluid near the wall. In this case, the use of a conjugate approach is required to correctly predict the near wall behaviour as well as the local non-uniform wall surface temperature. The effects of the fluid/wall thermal interaction are expected to reduce as the conduction characteristic time of the solid increases.

\section{Acknowledgments}

The research leading to these results has received funding through the People Programme (Marie Curie Actions) of the European Union's Seventh Framework Programme (FP7, 2007-2013) under the grant agreement No. FP7-290042 for the project COPA-GT. The authors thankfully acknowledge the computer resources, technical expertise and assistance provided by the Red Española de Supercomputación (RES).

\section{References}

[1] S. Jaure, F. Duchaine, G. Staffelbach, L. Gicquel, Massively parallel conjugate heat transfer methods relying on large eddy simulation applied to an aeronautical combustor, Comput. Sci. Disc. 6 (2013) 015008.

[2] R. S. Bunker, Gas turbine heat transfer: Ten remaining hot gas path challenges, J Turbomach. 129 (2007) 193-201.

[3] F. Duchaine, S. Mendez, F. Nicoud, A. Corpron, V. Moureau, T. Poinsot, Coupling heat transfer solvers and large eddy simulations for combustion applications, in: CTR Proc. Summer Program, 2008.

[4] C. Farhat, M. Lesoinne, Two efficient staggered algorithms for the serial and parallel solution of three-dimensional nonlinear transient aeroelastic problems, Comput. Methods Appl. Mech. Engrg. 182 (2000) 499-515.

[5] V. Garg, Heat transfer research on gas turbine airfoils at nasa grc, Int. J. Heat Fluid Fl. 23 (2002) 109-136.

[6] P. Ferrero, D. D'Ambrosio, A numerical method for conjugate heat transfer problems in hypersonic flows, AIAA J. (2008) 4247.

[7] F. Duchaine, S. Mendez, F. Nicoud, A. Corpron, V. Moureau, T. Poinsot, Conjugate heat transfer with large eddy simulation for gas turbine components, C. R. Mecanique 337 (2009) 550-561. 
[8] T. Craft, H. Iacovides, J. Yoon, Progress in the use of non-linear two-equation models in the computation of convective heat-transfer in impinging and separated flows, Flow Turb. Combust. 63 (2000) 59-80.

[9] A. Chatelain, F. Ducros, M. Olivier, Large eddy simulation of conjugate heattransfer using thermal wall-functions, ERCOFTAC Series 9 (2004) 307-314.

[10] N. Maheu, V. Moureau, P. Domingo, F. Duchaine, G. Balarac, Large-eddy simulations of flow and heat transfer around a low-mach number turbine blade, in: CTR Proc. Summer Program, 2012.

[11] D. Mira, X. Jiang, C. Moulinec, D. Emerson, Numerical investigation of the effects of fuel variability on the dynamics of syngas impinging jet flames, Fuel 103 (2013) 646-662.

[12] M. Hadziabic, K. Hanjalic, Vortical structures and heat transfer in a round impinging jet, J. Fluid Mech. 596 (2008) 221-260.

[13] L. F. L., T. Hallqvist, Numerical study of impinging jets with heat transfer-inlet conditions effects, AIAA (2009) 1578.

[14] T. Dairay, V. Fortune, E. Lamballais, L. Brizzi, Les of a turbulent jet impinging on a heated wall using high-order numerical schemes, Int. J. Heat Fluid Fl. 50 (2011) 177-187.

[15] A. Dewan, R. Dutta, B. Srinivasan, Recent trends in computation of turbulent jet impingement heat transfer, Heat Transfer Eng. 22 (2012) 447460.

[16] G. Lodato, L. Vervisch, P. Domingo, A compressible wall-adapting similarity mixed model for large-eddy simulation of the impinging round jet, Phys. Fluids 21 (2009) 035102.

[17] K. R. Dinesh, X. Jiang, J. A. van Oijen, Numerical simulation of hydrogen impinging jet flame using flamelet generated manifold reduction, Int. J. Hydrogen Energ. 37 (2012) 4502-4515.

[18] X. Jiang, K. Luo, L. de Goey, R. Bastiaans, J. van Oijen, Swirling and impinging effects in an annular nonpremixed jet flame, Flow Turb. Combust. 86 (2011) 63-88.

[19] D. Mira, X. Jiang, Numerical investigations of a hydrogen impinging flame with different finite-rate chemical kinetic mechanisms, Fuel 109 (2013) 285-296.

[20] P. Pantangi, A. Sadiki, J. Janicka, M. Mann, A. Dreizler, Les of premixed methane flame impinging on the wall using non-adiabatic flamelet generated manifold (fgm) approach, Flow Turb. Combust. 92 (2014) 805-836. 
[21] T. Poinsot, D. Veynante, Theoretical and Numerical Combustion, Ed. Edwards, 3rd Edition, 2012.

[22] F. Nicoud, F. Ducros, Subgrid-scale stress modelling based on the square of the velocity gradient, Flow Turb. Combust. 62 (1999) 183-200.

[23] D. Mira, X. Jiang, C. Moulinec, D. Emerson, Numerical assessment of subgrid scale models for scalar transport in large-eddy simulations of hydrogen-enriched fuels, Int. J. Hydrogen Energy 39 (2014) 7173-7189.

[24] R. K. Jaiman, X. Jiao, P. Geubelle, E. Loth, Conservative load transfer along curved fluidsolid interface with non-matching meshes, J. Comput. Phys. 218 (2006) 372-397.

[25] Y. Fournier, Parallel location and exchange, Tech. rep., Électricité de France (EDF) (2014).

[26] T. Mantel, F. Egolfopoulos, C. Bowman, A new methodology to determine kinetic parameters for one- and two-step chemical models, in: CTR Proc. Summer Program, 1996.

[27] O. Collin, F. Ducros, D. Veynante, T. Poinsot, A thickened flame model for large eddy simulations of turbulent premixed combustion, Phys. Fluids 12 (2000) $1843-1863$.

[28] L. Durand, A.Huber, W. Polifke, Implementation and validation of les models for inhomogeneously premixed turbulent combustion, in: Proc. European Combustion Meeting, Louvain-la-Neuve, Belgium, 2005.

[29] G. Houzeaux, J. Principe, A variational subgrid scale model for transient incompressible flows, Int. J. Comput. Fluid D. 22 (2008) 135-152.

[30] G. Houzeaux, R. Aubry, M. Vazquez, Extension of fractional step techniques for incompressible flows: the preconditioned orthomin (1) for the pressure schur complement, Comput. Fluids 40 (2011) 297313.

[31] R. Lohner, F. M. J. Cebral, R. Aubry, G. Houzeaux, Deflated preconditioned conjugate gradient solvers for the pressure-poisson equation: extensions and improvements, Int. J. Numer. Meth. Engn. 87 (2011) 2-14.

[32] G. Houzeaux, M. Vazquez, R. Aubry, J. Cela, A massively parallel fractional step solver for incompressible flows, J. Comput. Phys. 228 (2009) 63166332.

[33] R. Kee, G. Dixon-Lewis, J. Warnatz, M. Coltrin, J. Miller, A fortran computer code package for the evaluation of gas-phase multicomponent transport properties, Tech. Rep. SAND86-8246, Sandia National Laboratories (1986). 
[34] G. Smith, D. Golden, M. Frenklach, N. Moriarty, B. Eiteneer, M. Goldenberg, C. T. Bowman, R. Hanson, S. Song, W. Gardiner, V. Lissianski, Z. Qin, GRIMech 3.0 (1999). URL http://www .me.berkeley . edu/gri-mech/

[35] A. Chatelain, F. Ducros, O. Métais, Large Eddy Simulation of Conjugate HeatTransfer Using Thermal Wall-Functions, Direct and Large-Eddy Simulation V, Kluwer Academic Publisher, 2004.

[36] R. Jaiman, X. Jiao, P. Geubelle, E. Loth, Conservative load transfer along curved fluidsolid interface with non-matching meshes, J. Comput. Phys. 218 (2006) 373-397.

[37] B. Roe, R. Jaiman, A. Haselbacher, P. Geubelle, Combined interface boundary conditions method for coupled thermal simulations, Int. J. Numer. Meth. Fluids 57 (2008) 329-354.

[38] M. Tummers, J. Jacobse, S. Voorbrood, Turbulent flow in the near field of a round impinging jet, Int. J. Heat Mass Transfer 54 (2011) 4939-4948.

[39] N. Jarrin, S. Benhamadouche, D. Laurence, R. Prosser, A synthetic-eddymethod for generating inflow conditions for large-eddy simulations, Int. J. Heat Fluid Fl. 27 (2006) 585-593.

[40] T. Natarajan, J. Jewkes, R. Narayanaswamy, Y. Chung, A. Lucey, Reynolds averaged and large eddy computations of flow and heat transfer under round jet impingement, in: Proc. ASME, Chicago, Illinois, USA, 2014, p. V01AT09A009.

[41] F.Shum-Kivan, F. Duchaine, L. Gicquel, Large-eddy simulation and conjugate heat transfer in a round impinging jet, in: Proc. ASME Turbo Expo, Dusseldorf, Germany, 2014.

[42] N. Uddin, S. Neumann, B. Weigand, \{LES $\}$ simulations of an impinging jet: On the origin of the second peak in the nusselt number distribution, Int. J. Heat Mass Transfer 57 (2013) 356 - 368. 